\title{
Computing the Reverse Eccentric Connectivity Index for Certain Family of Nanocone and Fullerene Structures
}

\author{
Wei Gao ${ }^{1}$ and Mohammad Reza Farahani² \\ ${ }^{1}$ School of Information Science and Technology, Yunnan Normal University, Kunming 650500, China \\ ${ }^{2}$ Department of Applied Mathematics, Iran University of Science and Technology, Narmak, Tehran 16844, Iran \\ Correspondence should be addressed to Wei Gao; gaowei@ynnu.edu.cn
}

Received 3 February 2016; Accepted 13 March 2016

Academic Editor: Paresh Chandra Ray

Copyright (C) 2016 W. Gao and M. R. Farahani. This is an open access article distributed under the Creative Commons Attribution License, which permits unrestricted use, distribution, and reproduction in any medium, provided the original work is properly cited.

\begin{abstract}
A large number of previous works reveal that there exist strong connections between the chemical characteristics of chemical compounds and drugs (e.g., melting point and boiling point) and their topological structures. Chemical indices introduced on these molecular topological structures can help chemists and material and medical scientists to grasp its chemical reactivity, biological activity, and physical features better. Hence, the study of the topological indices on the material structure can make up the defect of experiments and provide the theoretical evidence in material engineering. In this paper, we determine the reverse eccentric connectivity index of one family of pentagonal carbon nanocones $\mathrm{PCN}_{5}[n]$ and three infinite families of fullerenes $C_{12 n+2}, C_{12 n+4}$, and $C_{18 n+10}$ based on graph analysis and computation derivation, and these results can offer the theoretical basis for material properties.
\end{abstract}

\section{Introduction}

With the rapid development of material manufacture techniques, a great number of new nanomaterials are discovered each year. It needs a large number of experiments to test the chemical properties of numerous new materials, which increases the workload of the researchers. Luckily, a large number of former chemical based experiments drew the conclusion that there is an intrinsical and inevitable connection between topology structure of chemical molecular and their chemical characteristics, biological features, and physical behaviors, like melting point, boiling point, and toxicity (see Wiener [1] and Katritzky et al. [2] for more details).

In chemistry graph theory setting, materials and other chemical compounds are represented as graphs: each vertex in graph expresses an atom of molecule structure; each edge represents a covalent bound between two atoms. Such a graph is called molecular graph which is denoted as $G=$ $(V(G), E(G)$ ), where $V(G)$ is the vertex (atom) set and $E(G)$ is the edge (chemical bond) set. All the (molecular) graphs discussed in this paper are no loop and multiple edge, that is, simple graphs. The notations and terminologies used in our paper but not defined can be attributed to Bondy and Murty [3].

The topological index defined on a molecule structure can be regarded as a nonempirical numerical quantity or a nonnegative score function which quantified the material structure and its branching pattern. Therefore, it can be used as a descriptor of the molecule under experiments and can be applied in several chemical engineering applications, such as QSPR/QSAR study. Several contributions on this field can be found in Yan et al. [4], Gao and Shi [5], and Gao and Wang $[6,7]$ for more details.

There are several indices introduced in chemical and pharmacy engineering and also used to test the properties of nanomaterials. The eccentricity ec $(u)$ of vertex $u \in V(G)$ is defined as the maximum distance between $u$ and any other vertex in $G$. Then, the eccentric connectivity index (ECI) of (molecular) graph $G$ is defined as

$$
\xi^{c}(G)=\sum_{v \in V(G)} \mathrm{ec}(v) d(v) .
$$

Ranjini and Lokesha [8] studied the eccentric connectivity index of the subdivision graph of the wheel graphs, tadpole 
graphs, and complete graphs. Morgan et al. [9] deduced the exact lower bound on $\xi^{c}(G)$ by means of order and presented the sharpness of this bound. An asymptotically tight upper bound was also inferred. Additionally, for trees of fixed vertex number and diameter, the precise upper and lower bounds are manifested. Hua and Das [10] considered the relationship between the Zagreb indices and eccentric connectivity index. De [11] raised the explicit generalized expressions for the eccentric connectivity index and its polynomial of the thorn graphs. Eskender and Vumar [12] computed the eccentric connectivity index and eccentric distance sum of generalized hierarchical product of graphs. Furthermore, the exact formulae for the eccentric connectivity index of $F$-sum graphs by means of certain invariants of the factors are also determined. Ilić and Gutman [13] presented that the broom has maximum $\xi^{c}(G)$ among trees with given maximum vertex degree, and the trees with minimum $\xi^{c}(G)$ are characterized as well. Iranmanesh and Hafezieh [14] calculated the eccentric connectivity index of several graph families. Dankelmann et al. [15] described the upper bound for eccentric connectivity index and some graphs are constructed which asymptotically attain such bound. Morgan et al. [16] showed that a known tight lower bound on the eccentric connectivity index for a tree, in view of vertex number and diameter, was also valid for a general graph. Rao and Lakshmi [17] yielded explicit formulas for eccentric connectivity index of phenylenic nanotubes.

Ediz [18] introduced a new distance-based molecular index called reverse eccentric connectivity index which was denoted as

$$
\operatorname{RE}^{c}(G)=\sum_{v \in V(G)} \frac{\mathrm{ec}(v)}{S(v)},
$$

where $S(v)$ is the sum of degrees of its neighborhoods; that is, $S(v)=\sum_{u \in N(v)} d(u)$. Nejati and Mehdi [19] determined the reverse eccentric connectivity index of one tetragonal carbon nanocone.

Although there have been several advances in eccentric connectivity related index of special molecular graphs, the research of reverse eccentric connectivity index for certain special chemical compound, nanophase materials, and drug structures is still largely limited. On the other hand, as critical and widespread chemical structures, pentagonal carbon nanocones and fullerenes are widely used in chemistry, biology, and medical and material science and frequently appeared in new chemical structures. For these important reasons, we present the exact expressions of reverse eccentric connectivity index for several pentagonal carbon nanocones and fullerenes structures.

In this paper, we mainly study the reverse eccentric connectivity index of pentagonal carbon nanocones $\mathrm{PCN}_{5}[n]$ in Section 2 and three infinite families of fullerenes $C_{12 n+2}$, $C_{12 n+4}$, and $C_{18 n+10}$ in Section 3.

\section{Reverse Eccentric Connectivity Index of Pentagonal Carbon Nanocone}

In this section, we aim to determine the reverse eccentric connectivity index of pentagonal carbon nanocone $\mathrm{PCN}_{5}[n]$

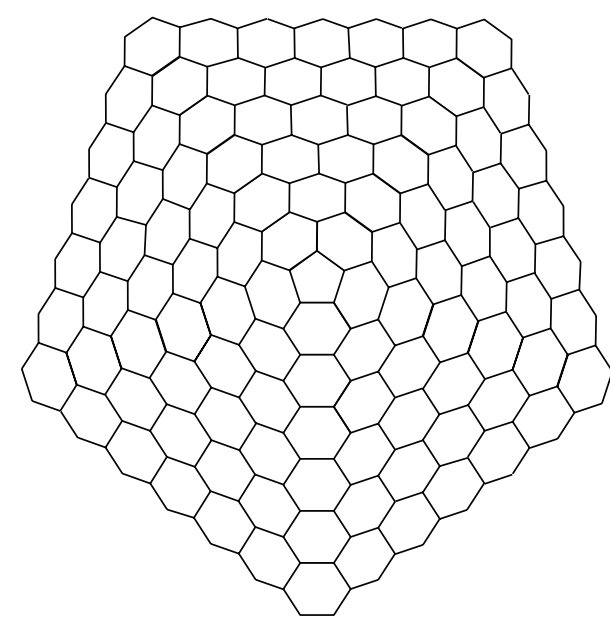

FIGURE 1: The molecular structure of pentagonal carbon nanocone $\mathrm{PCN}_{5}[n]$.

(see Figure 1 for its detailed structure) in terms of molecular structure analysis and an algebraic trick. After obtaining the exact expression of reverse eccentric connectivity index, we design a computer program using Java to determine its values for some fixed positive integer $n$.

It is easy to check that $\mathrm{PCN}_{5}[n]$ has $5 n^{2}$ and $5\left(3 n^{2}-n\right) / 2$ edges. For arbitrary vertex $v \in V\left(\mathrm{PCN}_{5}[n]\right)$, we have $2 n \leq$ $\mathrm{ec}(v) \leq 4 n-2$.

The main conclusion in this section is stated as follows.

Theorem 1. The reverse eccentric connectivity index of pentagonal carbon nanocone $\mathrm{PCN}_{5}[n]$ is stated as follows:

$$
{ }^{R E} \xi^{c}\left(P C N_{5}[n]\right)=\frac{50}{27} n^{3}+\frac{115}{126} n^{2}+\frac{17}{54} n-\frac{47}{63} .
$$

Proof. It can be seen that, in Figure 2, the whole pentagonal carbon nanocone $\mathrm{PCN}_{5}[n]$ can be divided into five equivalent partitions which are denoted by $S_{1}, S_{2}, \ldots, S_{5}$ and we have $\mathrm{PCN}_{5}[n]=\sum_{i=1}^{5} S_{i}$. The trick to analyze the structure is to focus on one part and then extend to the whole molecular structure.

Using the graph analysis, we know that the vertices in $S_{i}$ can be divided into $2 n$ classes (for convenience, we denote class 1 , class $2, \ldots$, class $2 n$ for these vertex classes) according to the value of ec $(v)$ and $S(v)$. The value of ec $(v)$ for vertex $v$ in class 1 and class 2 is $4 n-2$, and ec $(v)=4 n-i$ if vertex $v$ belongs to class $i$ for $3 \leq i \leq 2 n$. For vertex $v$ in class 1 , class 2 , and class 3 , the value of $S(v)$ is 5,6 , and 7, respectively. And, $S(v)=9$ if vertex $v$ belongs to class $i$, where $4 \leq i \leq 2 n$. Moreover, the number of vertices in class 1 and class 2 is 2 and $n-2$, respectively. The vertex number of class $i$ is $n-\lfloor(i-1) / 2\rfloor$ for $3 \leq i \leq 2 n$.

In order to simply and conveniently compute the reverse eccentric connectivity index, we design a program which is written using Java. The procedure is listed in Algorithm 1. 


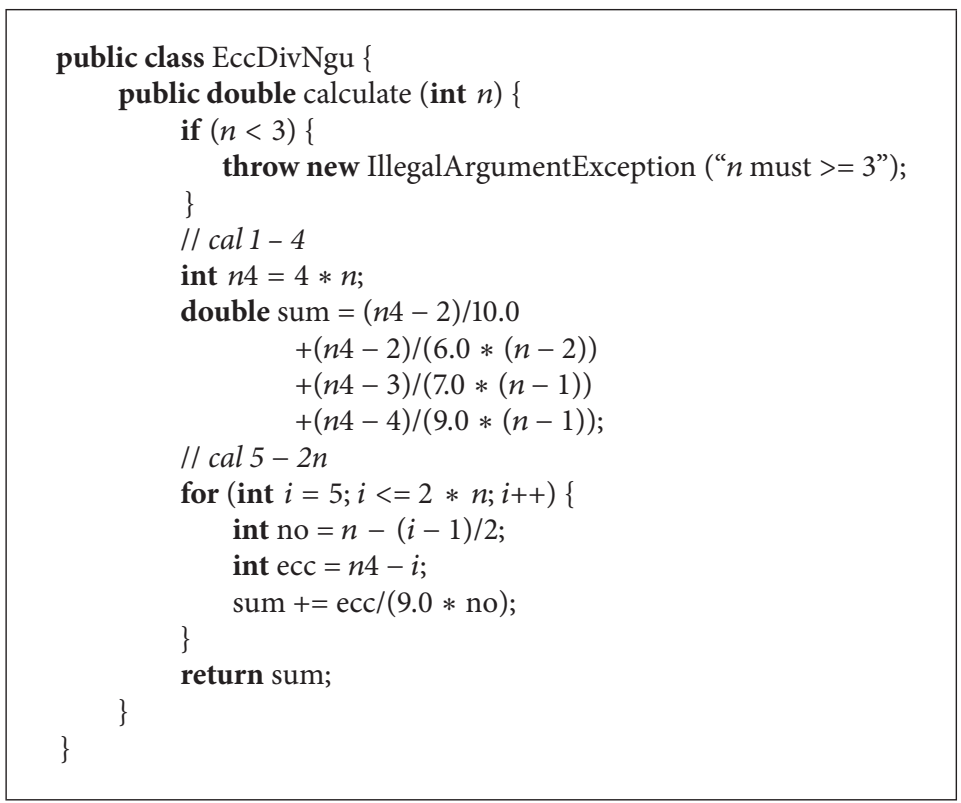

Algorithm 1

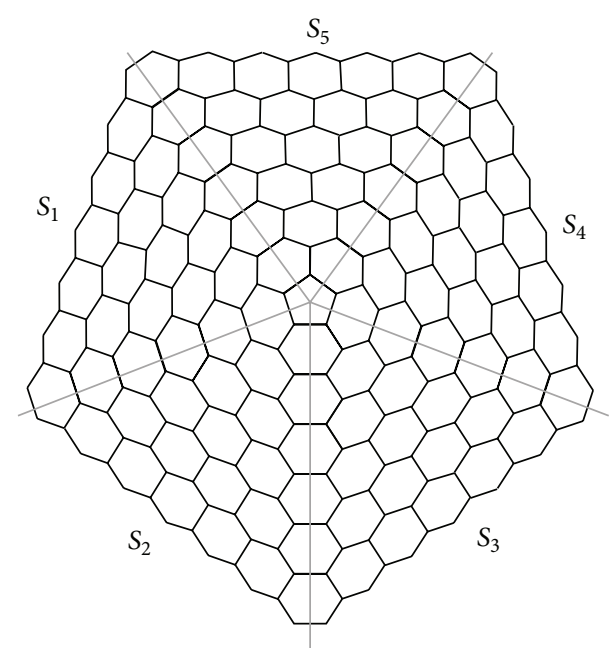

Figure 2: The partitions of pentagonal carbon nanocone $\mathrm{PCN}_{5}[n]$.

According to the vertex classification and distance computation, we have

$$
\begin{aligned}
& \mathrm{RE}^{c}\left(\mathrm{PCN}_{5}[n]\right)=\sum_{v \in V\left(\mathrm{PCN}_{5}[n]\right)} \frac{\mathrm{ec}(v)}{S(v)}=5 \sum_{v \in V\left(S_{1}\right)} \frac{\mathrm{ec}(v)}{S(v)} \\
& =5\left\{2 \frac{4 n-2}{5}+(n-2) \frac{4 n-2}{6}+(n-1) \frac{4 n-3}{7}\right. \\
& +(n-1) \frac{4 n-4}{9}+(n-3) \frac{4 n-5}{9}+(n-2) \frac{4 n-6}{9} \\
& +(n-3) \frac{4 n-7}{9}+\cdots+2 \frac{2 n+3}{9}+2 \frac{2 n+2}{9} \\
& \left.+\frac{2 n+1}{9}+\frac{2 n}{9}\right\}=5\left\{\frac{5 n+2}{30}(4 n-2)\right.
\end{aligned}
$$

$$
\begin{aligned}
& \left.+\frac{16}{63}(n-1)(4 n-3)+\frac{1}{9} \sum_{i=2}^{n-1}(n-i)(8 n-4 i-3)\right\} \\
& =5 \times\left(\frac{10}{27} n^{3}+\frac{23}{126} n^{2}+\frac{17}{270} n-\frac{47}{315}\right)=\frac{50}{27} n^{3} \\
& +\frac{115}{126} n^{2}+\frac{17}{54} n-\frac{47}{63} .
\end{aligned}
$$

Hence, the expected result is obtained.

We compute the reverse eccentric connectivity index of $\operatorname{PCN}_{5}[n]$ for $n \in\{1,2, \ldots, 10\}$, and the result data can be found in Table 1.

\section{Reverse Eccentric Connectivity Index of Fullerenes}

Fullerenes were found in 1985 by chemical experiments which can be regarded as zero-dimensional nanostructures. A number of carbon atoms for some classes of carboncage molecules are bonded in a nearly spherical configuration. Let $F$ be a fixed fullerene, and let $h, p, n$, and $m$ be the number of hexagons, pentagons, carbon atoms, and chemical bonds, respectively. In terms of structure analysis, it can be easily found that each atom lies in exactly three faces and each chemical bound (edge) lies in two faces. Hence, we infer that $n=(5 p+6 h) / 3$ is the number of atoms, $m=3 n / 2$ is the number of bounds (edges), and $f=p+h$ is the number of faces. By means of Euler formula, $n \mathrm{Cm}+f=2$, we yield $m=3 h+30, n=2 h+20$, and $p=12$. It reveals that this chemical structure consists of $n$ carbon atoms and contains 12 pentagonal faces and $n / 2-10$ hexagonal faces. See Prylutskyy et al. [20], Borisova et al. [21], Sugikawa et al. [22], Heumueller et al. [23], and Hendrickson et al. [24] for more 
TABLE 1: Some exceptional cases of reverse eccentric connectivity index of $\mathrm{PCN}_{5}[n]$.

\begin{tabular}{lr}
\hline$n$ & Reverse eccentric connectivity index \\
\hline 1 & $\mathrm{RE}^{c}\left(\mathrm{PCN}_{5}[1]\right)=\frac{7}{3}$ \\
2 & $\mathrm{RE}^{c}\left(\mathrm{PCN}_{5}[2]\right)=\frac{1165}{63}$ \\
3 & $\mathrm{RE}^{c}\left(\mathrm{PCN}_{5}[3]\right)=\frac{3680}{63}$ \\
4 & $\mathrm{RE}^{c}\left(\mathrm{PCN}_{5}[4]\right)=\frac{8419}{63}$ \\
5 & $\mathrm{RE}^{c}\left(\mathrm{PCN}_{5}[5]\right)=\frac{16073}{63}$ \\
6 & $\mathrm{RE}^{c}\left(\mathrm{PCN}_{5}[6]\right)=434$ \\
7 & $\mathrm{RE}^{c}\left(\mathrm{PCN}_{5}[7]\right)=\frac{42926}{63}$ \\
8 & $\mathrm{RE}^{c}\left(\mathrm{PCN}_{5}[8]\right)=\frac{3025}{3}$ \\
9 & $\mathrm{RE}^{c}\left(\mathrm{PCN}_{5}[9]\right)=\frac{89839}{63}$ \\
10 & $\mathrm{RE}^{c}\left(\mathrm{PCN}_{5}[10]\right)=\frac{40856}{21}$
\end{tabular}

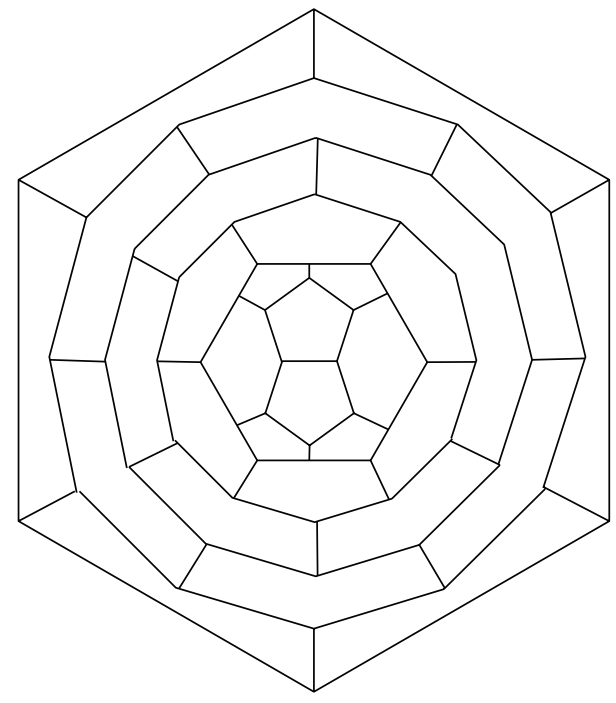

Figure 3: The molecular structure of fullerenes $C_{12 n+2}$.

details on the structure of fullerenes and their engineering applications.

The purpose of this section is to obtain the reverse eccentric connectivity index of three families of fullerenes. The first family is $C_{12 n+2}$ and its structure is presented in Figure 3.

The first result in this section is manifested below.

Theorem 2. The reverse eccentric connectivity index of fullerenes $C_{12 n+2}$ is

$$
{ }^{R E} \xi^{c}\left(C_{12 n+2}\right)=2 n^{2}+\frac{28}{9} n .
$$

TABLE 2: Some exceptional cases of reverse eccentric connectivity index of fullerenes $C_{12 n+2}$.

\begin{tabular}{|c|c|}
\hline$n$ & Reverse eccentric connectivity index \\
\hline 1 & $\mathrm{RE}^{\mathrm{C}}\left(C_{14}\right)=\frac{46}{9}$ \\
\hline 2 & $\mathrm{RE}^{c}\left(C_{26}\right)=\frac{128}{9}$ \\
\hline 3 & ${ }^{\mathrm{RE}} \xi^{c}\left(C_{38}\right)=\frac{82}{3}$ \\
\hline 4 & ${ }^{\mathrm{RE}} \xi^{c}\left(C_{50}\right)=\frac{400}{9}$ \\
\hline 5 & $\mathrm{RE}_{\xi^{c}}\left(C_{62}\right)=\frac{590}{9}$ \\
\hline 6 & $\mathrm{RE}^{c}\left(C_{74}\right)=\frac{272}{3}$ \\
\hline 7 & ${ }^{\mathrm{RE}} \xi^{c}\left(C_{86}\right)=\frac{1078}{9}$ \\
\hline 8 & ${ }^{\mathrm{RE}} \xi^{c}\left(C_{98}\right)=\frac{1376}{9}$ \\
\hline 9 & ${ }^{\mathrm{RE}} \xi^{c}\left(C_{110}\right)=190$ \\
\hline 10 & ${ }^{\mathrm{RE}} \xi^{c}\left(C_{122}\right)=\frac{2080}{9}$ \\
\hline
\end{tabular}

Proof. According to the structure of fullerenes $C_{12 n+2}$, we see that $S(v)=9$ for any $v \in V\left(C_{12 n+2}\right)$. By the value of ec $(v)$, all the vertices can be divided into three classes. There are 8 vertices and 6 vertices in class 1 and class 2 , and their ec $(v)$ are $2 n$ and $n$, respectively. For the last vertex class, we have $\mathrm{ec}(v)=n+i$ for $i \in\{1,2, \ldots, n\}$, and there are 12 vertices for each $i$. Thus, in view of the definition of reverse eccentric connectivity index, we have

$$
\begin{aligned}
\mathrm{RE}_{\xi^{c}}\left(C_{12 n+2}\right) & =6 \cdot \frac{n}{9}+8 \cdot \frac{2 n}{9}+12 \sum_{i=1}^{n} \frac{n+i}{9} \\
& =2 n^{2}+\frac{28}{9} n .
\end{aligned}
$$

We complete the proof.

As the former section, we list the value of ${ }^{\mathrm{RE}} \xi^{c}\left(C_{12 n+2}\right)$ for $n \in\{1,2, \ldots, 10\}$ in Table 2 .

The second family of fullerenes we discussed here is $C_{12 n+4}$ which is presented in Figure 4 for its structure.

The conclusion for this family of fullerenes is stated as follows.

Theorem 3. The reverse eccentric connectivity index of fullerenes $C_{12 n+4}$ is

$$
{ }^{R E} \xi^{c}\left(C_{12 n+4}\right)=2 n^{2}+\frac{38}{9} n+\frac{16}{9} .
$$

Proof. By means of the structure of fullerenes $C_{12 n+4}$, we ensure that $S(v)=9$ for arbitrary $v \in V\left(C_{12 n+4}\right)$. According to the value of ec $(v)$, the set $V\left(C_{12 n+4}\right)$ can be divided into two classes. The first class only has four vertices and ec $(v)=2 n+1$. 


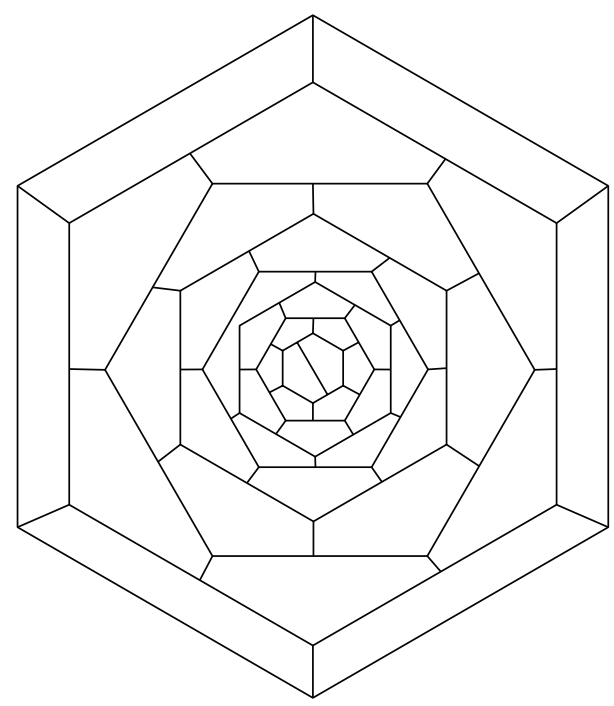

Figure 4: The molecular structure of fullerenes $C_{12 n+4}$.

TABLE 3: Some exceptional cases of reverse eccentric connectivity index of fullerenes $C_{12 n+4}$.

\begin{tabular}{|c|c|}
\hline$n$ & Reverse eccentric connectivity index \\
\hline 1 & $\mathrm{RE}_{\xi^{c}}\left(C_{16}\right)=8$ \\
\hline 2 & $\mathrm{RE}^{c}\left(C_{28}\right)=\frac{164}{9}$ \\
\hline 3 & ${ }^{\mathrm{RE}} \xi^{c}\left(C_{40}\right)=\frac{292}{9}$ \\
\hline 4 & ${ }^{\mathrm{RE}} \xi^{c}\left(C_{52}\right)=\frac{152}{3}$ \\
\hline 5 & $\mathrm{RE}_{\xi^{c}}\left(C_{64}\right)=\frac{656}{9}$ \\
\hline 6 & $\mathrm{RE}_{\xi^{c}}\left(C_{76}\right)=\frac{892}{9}$ \\
\hline 7 & $\mathrm{RE}_{\xi^{c}}\left(C_{88}\right)=\frac{388}{3}$ \\
\hline 8 & ${ }^{R E} \xi^{c}\left(C_{100}\right)=\frac{1472}{9}$ \\
\hline 9 & ${ }^{\mathrm{RE}} \xi^{c}\left(C_{112}\right)=\frac{1816}{9}$ \\
\hline 10 & ${ }^{R E} \xi^{c}\left(C_{124}\right)=244$ \\
\hline
\end{tabular}

The second class can be further divided into $n+1$ subclasses (each subclass has 12 vertices), and for $i$ th subclass we get $\mathrm{ec}(v)=n+i$, where $i \in\{1,2, \ldots, n+1\}$. Hence, using the definition of reverse eccentric connectivity index, we infer

$$
\begin{aligned}
\mathrm{RE}_{\xi^{c}}\left(C_{12 n+4}\right) & =4 \cdot \frac{2 n+1}{9}+12 \sum_{i=1}^{n+1} \frac{n+i}{9} \\
& =2 n^{2}+\frac{38}{9} n+\frac{16}{9} .
\end{aligned}
$$

Therefore, the expected conclusion is obtained.

Again, we list the exceptional cases for $n \in\{1,2, \ldots, 10\}$ in Table 3.

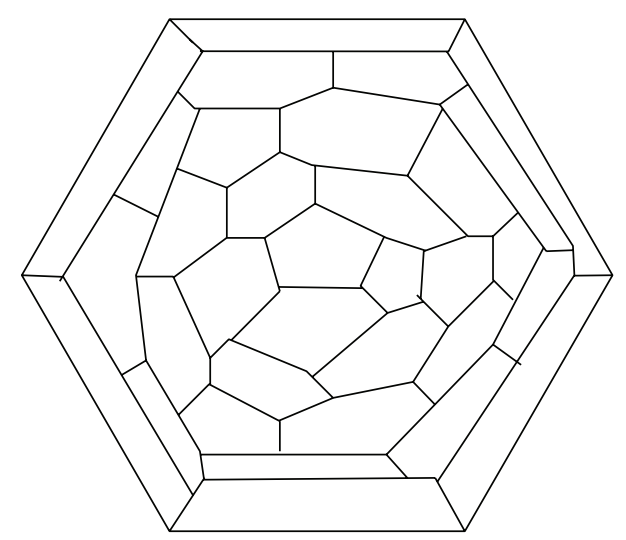

Figure 5: The molecular structure of fullerenes $C_{18 n+10}$.

The last family of fullerenes we study in this paper is $C_{18 n+10}$. See Figure 5 for its detailed structure.

The last formulation in our paper is presented as follows.

Theorem 4 . The reverse eccentric connectivity index of fullerenes $C_{18 n+10}$ is

$$
{ }^{R E} \xi^{c}\left(C_{18 n+10}\right)=3 n^{2}+\frac{47}{9} n+4 .
$$

Proof. The technology used here is similar to the trick used in former two theorems. First, we deduce $S(v)=9$ for any vertex $v$ in $C_{18 n+10}$. Then, we divide the vertex set of fullerenes $C_{18 n+10}$ into five classes according to the value of ec $(v)$. The values of ec $(v)$ for vertex in first four classes are $2 n, 2 n+$ $1,2 n+2$, and $2 n+3$, respectively. And, the corresponding numbers of vertices for first four classes are 15, 15, 9, and 7, respectively. The last class can be divided into $n-2$ subclasses such that $\mathrm{ec}(v)=n+i$ for $i \in\{2, \ldots, n-1\}$, and there are 18 vertices in each subclass of the last class. Finally, by the definition of reverse eccentric connectivity index, we deduce

$$
\begin{aligned}
\mathrm{RE}^{c}\left(C_{18 n+10}\right)= & 9 \cdot \frac{2 n+2}{9}+7 \cdot \frac{2 n+3}{9}+15 \\
& \cdot \frac{2 n+(2 n+1)}{9}+18 \sum_{i=2}^{n-1} \frac{n+i}{9} \\
= & 3 n^{2}+\frac{47}{9} n+4 .
\end{aligned}
$$

Hence, we verify the conclusion.

Table 4 listed as follows manifests the exceptional cases of $\mathrm{RE}^{c}{ }^{c}\left(C_{18 n+10}\right)$ for $n \in\{1,2, \ldots, 10\}$.

In this paper, in terms of graph analysis, distance computation, and vertex dividing, we give the exact expression of reverse eccentric connectivity index for four special chemical molecular structures. The conclusions yielded in our paper make up the shortage of experiments and provide a theoretical basis for scientific research. From this point of view, these results illustrate the promising application prospects in chemical, material, and pharmacy engineering. 
TABLE 4: Some exceptional cases of reverse eccentric connectivity index of fullerene $C_{18 n+10}$.

\begin{tabular}{lr}
\hline$n$ & Reverse eccentric connectivity index \\
\hline 1 & $\mathrm{RE}^{c}\left(C_{28}\right)=\frac{110}{9}$ \\
2 & $\mathrm{RE}^{c}\left(C_{46}\right)=\frac{238}{9}$ \\
3 & $\mathrm{RE}^{c}\left(C_{64}\right)=\frac{140}{3}$ \\
4 & $\mathrm{RE}^{c}\left(C_{82}\right)=\frac{656}{9}$ \\
5 & $\mathrm{RE}^{c}\left(C_{100}\right)=\frac{946}{9}$ \\
6 & $\mathrm{RE}^{c}\left(C_{118}\right)=\frac{430}{3}$ \\
7 & $\mathrm{RE}^{c}\left(C_{136}\right)=\frac{1688}{9}$ \\
8 & $\mathrm{RE}^{c}\left(C_{154}\right)=\frac{2140}{9}$ \\
9 & $\mathrm{RE}^{c}\left(C_{172}\right)=294$ \\
10 & $\mathrm{RE}_{\xi^{c}}\left(C_{190}\right)=\frac{3206}{9}$ \\
\hline
\end{tabular}

\section{Competing Interests}

The authors declare that there is no conflict of interests regarding the publication of this paper.

\section{Acknowledgments}

The research is partially supported by NSFC (nos. 11401519, 11371328 , and 11471293).

\section{References}

[1] H. J. Wiener, "Structural determination of paraffin boiling points," Journal of the American Chemical Society, vol. 69, no. 1, pp. 17-20, 1947.

[2] A. R. Katritzky, R. Jain, A. Lomaka, R. Petrukhin, U. Maran, and M. Karelson, "Perspective on the relationship between melting points and chemical structure," Crystal Growth \& Design, vol. 1, no. 4, pp. 261-265, 2001.

[3] J. A. Bondy and U. S. R. Murty, Graph Theory, Springer, Berlin, Germany, 2008.

[4] L. Yan, W. Gao, and J. S. Li, "General harmonic index and general sum connectivity index of polyomino chains and nanotubes," Journal of Computational and Theoretical Nanoscience, vol. 12, no. 10, pp. 3940-3944, 2015.

[5] W. Gao and L. Shi, "Wiener index of gear fan graph and gear wheel graph," Asian Journal of Chemistry, vol. 26, no. 11, pp. 3397-3400, 2014.

[6] W. Gao and W. F. Wang, "Second atom-bond connectivity index of special chemical molecular structures," Journal of Chemistry, vol. 2014, Article ID 906254, 8 pages, 2014.

[7] W. Gao and W. F. Wang, "The vertex version of weighted Wiener number for bicyclic molecular structures," Computational and
Mathematical Methods in Medicine, vol. 2015, Article ID 418106 10 pages, 2015.

[8] P. S. Ranjini and V. Lokesha, "Eccentric connectivity index, hyper and reverse-wiener indices of the subdivision graph," General Mathematics Notes, vol. 2, pp. 34-46, 2011.

[9] M. J. Morgan, S. Mukwembi, and H. C. Swart, "On the eccentric connectivity index of a graph," Discrete Mathematics, vol. 311, no. 13, pp. 1229-1234, 2011.

[10] H. Hua and K. C. Das, "The relationship between the eccentric connectivity index and Zagreb indices," Discrete Applied Mathematics, vol. 161, no. 16-17, pp. 2480-2491, 2013.

[11] N. De, "On eccentric connectivity index and polynomial of thorn graph," Applied Mathematics, vol. 3, no. 8, pp. 931-934, 2012.

[12] B. Eskender and E. Vumar, "Eccentric connectivity index and eccentric distance sum of some graph operations," Transactions on Combinatorics, vol. 2, no. 1, pp. 103-111, 2013.

[13] A. Ilić and I. Gutman, "Eccentric connectivity index of chemical trees," MATCH Communications in Mathematical and in Computer Chemistry, vol. 65, no. 3, pp. 731-744, 2011.

[14] M. Iranmanesh and R. Hafezieh, "The eccentric connectivity index of some special graphs," Iranian Journal of Mathematical Chemistry, vol. 2, pp. 61-65, 2011.

[15] P. Dankelmann, M. J. Morgan, S. Mukwembi, and H. C. Swart, "On the eccentric connectivity index and Wiener index of a graph," Quaestiones Mathematicae, vol. 37, no. 1, pp. 39-47, 2014.

[16] M. J. Morgan, S. Mukwembi, and H. C. Swart, "A lower bound on the eccentric connectivity index of a graph," Discrete Applied Mathematics, vol. 160, no. 3, pp. 248-258, 2012.

[17] N. P. Rao and K. L. Lakshmi, "Eccentric connectivity index of V-phenylenic nanotubes," Digest Journal of Nanomaterials and Biostructures, vol. 6, no. 1, pp. 81-87, 2010.

[18] S. Ediz, "Reverse eccentric connectivity index," Optoelectronics and Advanced Materials-Rapid Communications, vol. 6, no. 5-6, pp. 664-667, 2012.

[19] A. Nejati and A. Mehdi, "On reverse eccentric connectivity index of one tetragonal carbon nanocones," Journal of Theoretical and Computational Science, vol. 1, no. 3, Article ID 1000115, 2014.

[20] Y. Prylutskyy, A. Bychko, V. Sokolova et al., "Interaction of C60 fullerene complexed to doxorubicin with model bilipid membranes and its uptake by HeLa cells," Materials Science and Engineering C: Materials for Biological Applications, vol. 59, pp. 398-403, 2016

[21] P. A. Borisova, M. S. Blanter, V. V. Brazhkin et al., "Interaction of amorphous fullerene $\mathrm{C}_{60}$ with austenite $\mathrm{Fe}-\mathrm{Ni}$ alloy at high temperatures and pressures," Journal of Alloys and Compounds, vol. 656, pp. 383-388, 2016.

[22] K. Sugikawa, A. Kubo, and A. Ikeda, "pH-responsive nanogels containing fullerenes: synthesis via a fullerene exchange method and photoactivity," Chemistry Letters, vol. 45, no. 1, pp. 60-62, 2016.

[23] T. Heumueller, W. R. Mateker, A. Distler et al., "Morphological and electrical control of fullerene dimerization determines organic photovoltaic stability," Energy Environ. Sci., vol. 9, no. 1, pp. 247-256, 2016.

[24] O. D. Hendrickson, N. I. Smirnova, A. V. Zherdev, P. G. Sveshnikov, and B. B. Dzantiev, "Competitive photometric enzyme immunoassay for fullerene C-60 and its derivatives using a fullerene conjugated to horseradish peroxidase," Microchimica Acta, vol. 183, no. 1, pp. 211-217, 2016. 

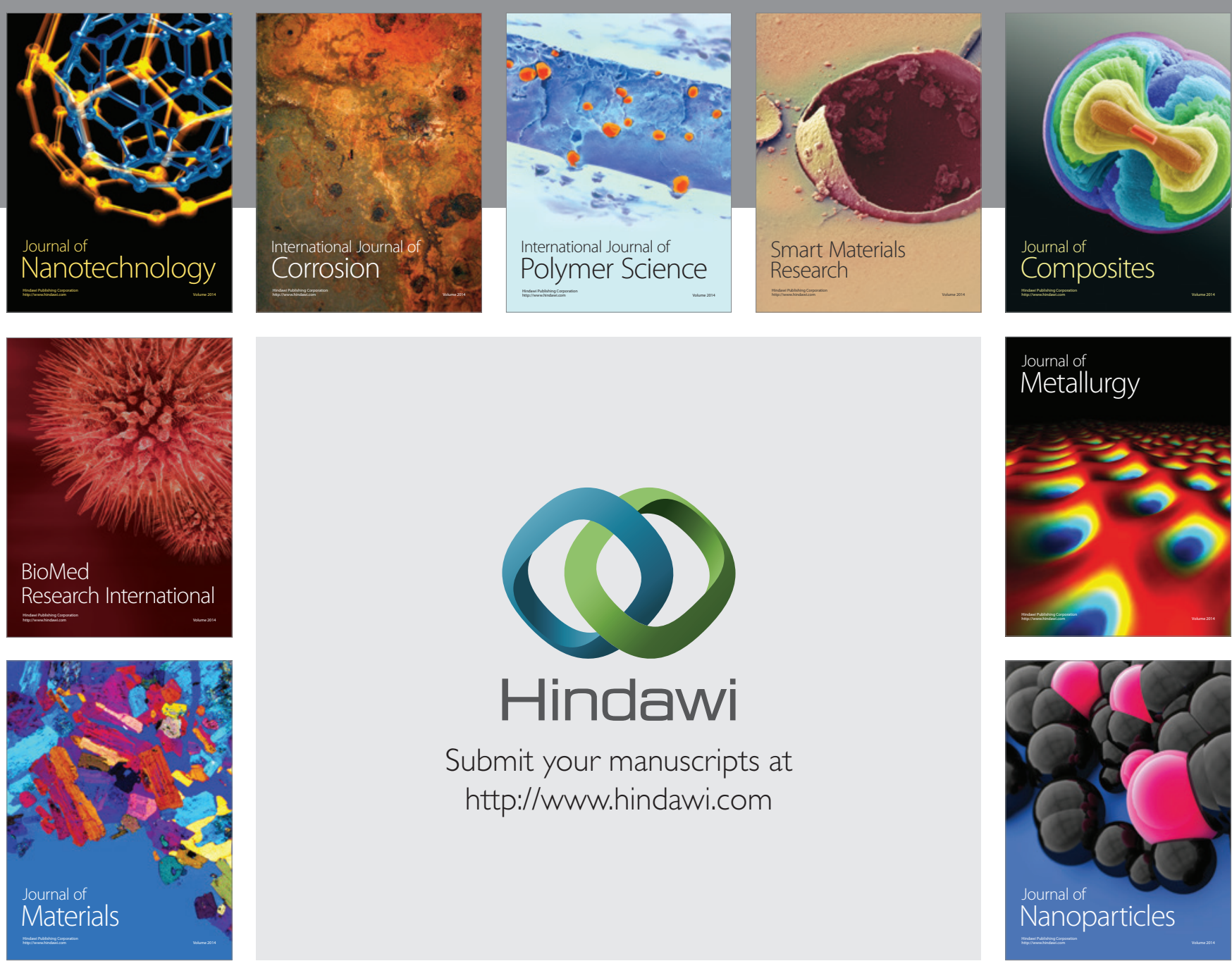

\section{Hindawi}

Submit your manuscripts at

http://www.hindawi.com

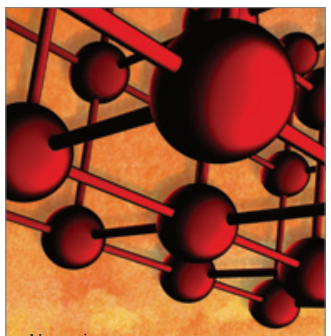

Materials Science and Engineering
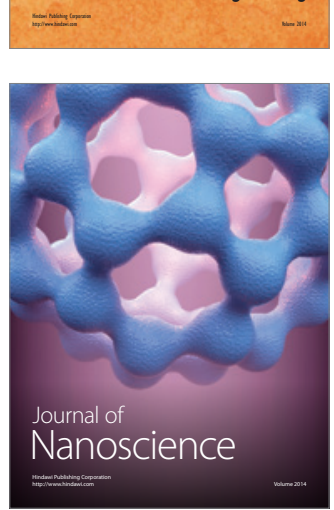
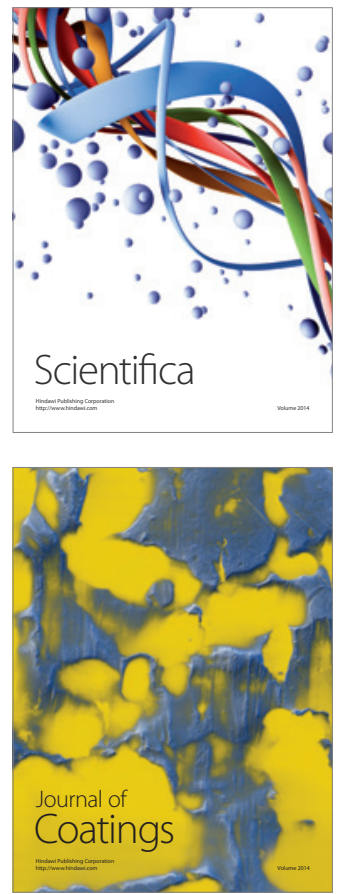
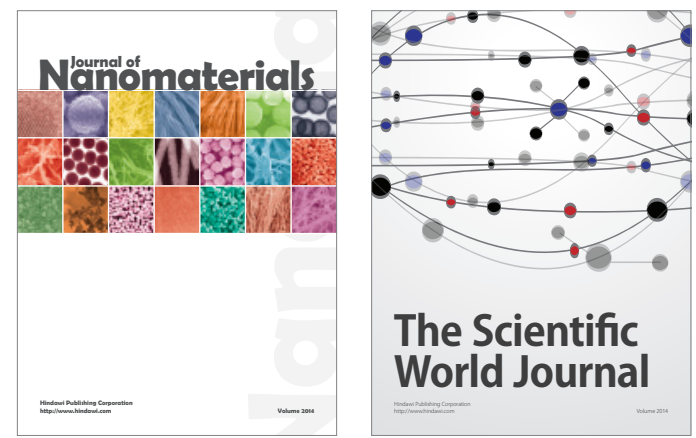

The Scientific World Journal
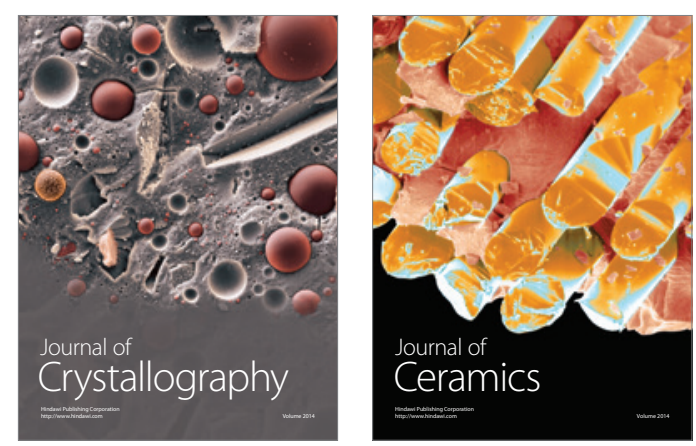
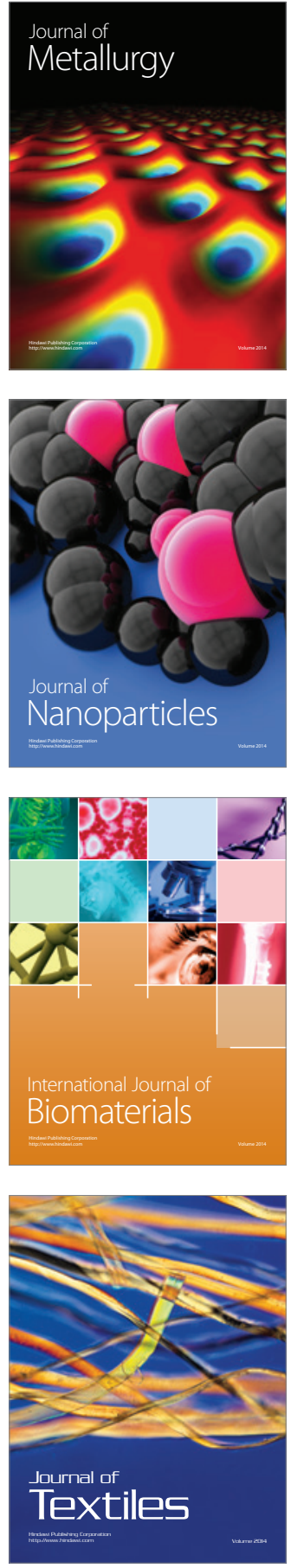